\title{
O QUE SE PUBLICA SOBRE COMPORTAMENTO DO CONSUMIDOR NO BRASIL, AFINAL?
}

\author{
What is published on Consumer \\ Behavior in Brazil, after all?
}

\author{
Marcelo de Rezende Pinto ${ }^{1}$ \\ José Edson Lara ${ }^{2}$
}

\section{Resumo}

Este artigo tem o objetivo de fazer uma análise dos artigos publicados sobre o tema comportamento do consumidor, utilizando os principais veículos de divulgação da produção acadêmica em marketing no Brasil de 1997 a 2006. Baseado no guia proposto por Hoppen, Moreau e Lapointe (1997), analisaram-se 238 artigos, por meio da verificação da utilização de elementos básicos de uma investigação, concentrando-se nas estratégias e metodologias de pesquisa. Após as análises referentes aos tipos de artigo, abordagem, natureza da pesquisa, metodologias e segmento de consumidores pesquisados, o estudo foi conduzido dividindo-se os artigos em três categorias: artigos do tipo survey, artigos do tipo experimental e aqueles baseados em pesquisas qualitativas. Pode-se afirmar que os principais resultados do trabalho foram: a constatação da predominância de trabalhos com base empírica, de abordagem positivista e da utilização de métodos e técnicas quantitativistas tradicionais de pesquisa e coleta de dados. Ressalta-se também pouca preocupação dos pesquisadores com alguns aspectos relevantes da qualidade metodológica. O artigo apresenta, ainda, reflexões sobre novas metodologias que poderiam ser utilizadas para se pesquisar o consumidor e pontos que contribuiriam para a consolidação da temática no Brasil.

Palavras-chave: Comportamento do Consumidor; Metodologias de Pesquisa; Surveys; Pesquisas Experimentais; Pesquisas Qualitativas.

${ }^{1}$ Mestre e doutorando em Administração pelo CEPEAD/UFMG. Professor da Pontifícia Universidade Católica de Minas Gerais - PUC Minas,. E-mail: marcrez@hotmail.com

${ }^{2}$ Phd em Administração pela Universitat Autònoma de Barcelona, Espanha. Professsor Adjunto do CEPEAD/UFMG. E-mail: jedson@face.ufmg.br. 


\begin{abstract}
This article aims to do an analysis of published articles on the topic behavior of the consumer using the principal vehicle for the dissemination of academic production in marketing in Brazil from 1997 to 2006. Based on the guidance proposed by Hoppen, Moreau and Lapointe (1997), were analyzed 238 articles by checking the use of basic elements of an investigation, concentrating on strategies and methodologies of research. After the tests on type of article, approach, nature of research, methodologies and searched segment of consumers, the study was conducted dividing up the articles into three categories: articles such survey, articles such trial and those based on qualitative research. It can be said that the main results of the study were: the finding of the preponderance of empirical work on the basis of positivist approach and use of traditional methods and techniques of quantitative research and data collection. It is important to observe a little concern of researchers with some relevant aspects of methodological quality. The article also presents ideas on new approaches that could be used to search the consumer and points that can contribute to the consolidation of the theme in Brazil.
\end{abstract}

Keywords: Consumer behavior; Research Methodology; Surveys; Experimental Research; Qualitative Research..

\title{
1 Introdução
}

A elaboração de trabalhos com o intuito de se fazer levantamento sobre a evolução de uma área da Administração ou um campo de estudo específico não é nova, até mesmo no Brasil. Um dos primeiros trabalhos publicados no Brasil com esse objetivo foi o de Machadoda-Silva, Cunha e Amboni (1990). Esses autores avaliaram os artigos da área de Organizações, percebendo uma fragilidade teórico-metodológica e um predomínio da abordagem funcionalista. A partir daí, outros estudos buscaram avaliar a construção do conhecimento nacional na área de Organizações, entre eles, Bertero e Keinert (1994), Vergara e Carvalho Jr. (1995), Rodrigues e Carrieri (2000). É possível também identificar trabalhos desse tipo que foram conduzidos em outras áreas da Administração: Leal, Oliveira e Soluri (2003) na área de Finanças, Hoppen e Meirelles (2005) em Tecnologia da Informação e Tonelli et al. (2003) na área de Recursos Humanos.

$\mathrm{Na}$ área de marketing, alguns trabalhos vêm tentando construir um corpo de conhecimento acerca da evolução dos estudos brasileiros. Um dos estudos desse tipo foi conduzido por Perin et al. (2000), com base nos artigos publicados na década de 1990. Os autores chegaram à conclusão de que as análises de pesquisas empíricas do tipo survey apresentam qualidade no mínimo questionável.

Contudo, um autor que realizou um amplo balanço sobre os estudos de marketing foi Vieira (1998, 1999, 2000). Esse autor, em 1998, ao estudar os artigos de marketing, publicados no Encontro Nacional dos Programas de Pós-graduação em Administração (Enanpad) na década de 1990, constatou uma concentração de pesquisas em Comportamento do Consumidor, Estratégias de Mercado, Marketing de Serviços e Sistemas de Informação de Pesquisa de Marketing. Já em 2000, ao analisar 272 artigos publicados no Enanpad, a Revista de Administração da USP (RAUSP) e a Revista de Administração de Empresas (RAE), Vieira (2000) verificou que houve um nítido direcionamento dos estudos no sentido de se conhecer melhor o consumidor brasileiro. 
Corroborando a constatação de Vieira (2000), o estudo publicado por Faria et al. (2006) também concluiu que a área de comportamento do consumidor teve o maior número de publicações durante o período de 2000 a 2005. Os autores visualizaram que houve um salto considerável de participação de estudos em comportamento do consumidor no período pesquisado.

Porém, em consulta aos Anais do Enanpad, além de pesquisas na Revista de Administração Contemporânea (RAC), na Revista de Administração de Empresas (RAE) e na Revista de Administração (RAUSP), no período de 1997 a 2006, não foi encontrado nenhum trabalho com o escopo de avaliar a produção científica com foco específico no tema do comportamento do consumidor.

Dessa forma, a partir da percepção de uma falta de estudos nessa área, surgiu o interesse em conduzir uma investigação com o objetivo de fazer um levantamento dos artigos sobre o tema comportamento do consumidor, utilizando os principais veículos de divulgação da produção acadêmica em marketing no Brasil: os anais do Enanpad e os periódicos das revistas RAE, RAC e RAUSP. Foram analisadas essas publicações nos últimos 10 anos, ou seja, de 1997 a 2006. A justificativa do estudo reside na importância de se estabelecer um "retrato" mais bem delineado para, dessa forma, direcionar as estratégias e temas estudados em pesquisas futuras, contribuindo para a consolidação dessa importante área de conhecimento do marketing.

Este trabalho está estruturado da seguinte forma: de início, discute-se o comportamento do consumidor como área de pesquisa de marketing, utilizando-se a análise das várias teorias e escolas de pensamento do marketing elaboradas por Sheth, Gardner e Garrett (1988). Achou-se conveniente incluir uma discussão sucinta sobre a pesquisa do consumidor e a evolução dessa área de pesquisa nas últimas décadas. Em seguida, são apresentados os procedimentos metodológicos adotados para fazer o levantamento. Após, são exibidos e discutidos os resultados. Por fim, as considerações finais do estudo são delineadas com reflexões sobre novas metodologias que poderiam ser utilizadas para se pesquisar o consumidor e pontos que contribuiriam para a consolidação da área no Brasil.

\section{O comportamento do consumidor como área da pesquisa no marketing}

Como o objetivo do trabalho é fazer um mapeamento das pesquisas na área do comportamento do consumidor nos últimos anos, optou-se por incluir, no referencial teórico, discussões acerca do tema. Sendo assim, este tópico visa a descrever a classificação proposta por Sheth, Gardner e Garrett (1988) para as várias teorias e escolas de marketing existentes para subsidiar a discussão de uma das escolas do pensamento: a do comportamento do consumidor, que é a base deste estudo.

Para denotar as similaridades entre as várias escolas de pensamento de marketing, Sheth, Gardner e Garrett (1988) propuseram uma matriz 2 X 2, que será utilizada para classificar as várias teorias e permitir uma compreensão entre as várias escolas de pensamento em termos de seus valores, orientações e filosofias básicas de motivação e comportamento humano.

O fundamento para essa classificação são as duas dimensões de perspectiva interativo X não-interativo e as duas dimensões de perspectiva econômica X não-econômica. A dimensão interativa $X$ não-interativa baseia-se em questões sobre o papel do marketing e seus objetivos. As escolas de pensamento baseadas em processos interativos incorporam o conceito de balanço de poder entre vendedores e compradores no mercado. Em contraste, isso não é assumido por escolas de pensamento que se baseiam na perspectiva não-interativa. Já a segunda dimensão se foca na orientação econômica $X$ não-econômica das teorias e é utilizada para enfatizar 
as diferentes abordagens para atingir os objetivos de marketing, assim como os pontos de vista de vendedores e compradores. As escolas de perspectiva econômica adotaram uma forte explicação econômica nas ações dos atores de marketing, enquanto a perspectiva nãoeconômica apóia-se no argumento de que as ações de produtores, membros dos canais de marketing e consumidores poderiam não ser explicadas somente por análises econômicas (SHETH, GARDNER e GARRETT, 1988). A matriz pode ser visualizada no Quadro 1.

De acordo com esses autores, as escolas de perspectiva não-interativa e econômica são aquelas que emergiram quando o marketing se divorciou da economia. Essas escolas têm grande relevância para a análise e prática do marketing moderno. As três escolas de abordagens interativa e econômica surgiram uma década depois das escolas de perspectiva não-interativa e econômica e demonstram visões mais avançadas e sofisticadas das atividades de marketing. Já as escolas baseadas em perspectivas não-interativa e não-econômica se desenvolveram durante os anos das décadas de 1960 e 1970 e representam uma grande mudança na orientação do marketing, visto que houve a emergência do interesse em influências comportamentais, sociais e psicológicas na disciplina. Por fim, o último grupo de escolas que utilizam a orientação interativa e não-econômica emergiram mais recentemente. As integrantes dessa perspectiva baseiam-se mais nas ciências comportamentais do que nas ciências econômicas e tanto compradores como vendedores são utilizados para analisar e entender as transações de mercado.

\begin{tabular}{|l|c|c|}
\hline & PERSPECTIVA NÃO-INTERATIVA & PeRSPECTIVA INTERATIVA \\
\hline PERSPECTIVA & Commodities & Institucional \\
ECONOMMICA & Funcional & Funcionalista \\
& Regional & Gerencial \\
\hline PERSPECTIVA & Comportamento do consumidor & Dinâmica organizacional \\
NÃO-ECONÔMICA & Ativista & Sistemas \\
& Macromarketing & Troca social \\
\hline
\end{tabular}

Fonte: Sheth, Gardner e Garrett (1988).

Quadro 1 - Classificação das escolas de marketing.

Com base nessa classificação, pode-se afirmar, portanto, que a escola do comportamento do consumidor nas perspectivas não-interativa e não-econômica será a utilizada no presente estudo. Essa escola, segundo Sheth, Gardner e Garrett (1988), representa uma mudança significativa na história do pensamento de marketing. A análise desses autores sugere duas razões para a rápida evolução e alta popularidade da escola de comportamento do consumidor: (1) a emergência do conceito de marketing e (2) o campo de conhecimento estabelecido na ciência comportamental.

Com relação à primeira razão, é lícito enfatizar que, depois da II Guerra Mundial, a economia americana começou a mudar de uma economia centrada nos vendedores para uma economia centrada nos compradores. A extraordinária capacidade de produção tinha gerado um excedente e começava a se perceber uma dificuldade para vender o que era produzido. Dessa forma, vários autores começaram a focar esforços no entendimento de como o consumidor deveria ser conquistado para fazer frente à grande concorrência.

No tocante ao campo de conhecimento estabelecido na ciência comportamental, havia também uma crescente realização de um considerável corpo de conhecimento oriundo das ciências comportamentais que poderia ser útil para funções de negócios, especialmente o marketing. Assim, conhecimentos da Antropologia, da Psicologia Cognitiva, entre outros, passaram a contribuir para os estudos do comportamento do consumidor.

Pode-se afirmar que os primeiros estudos relacionados ao comportamento 
do consumidor datam de meados da década de 1950. A partir daí, a área vem evoluindo, incorporando novas subáreas e campos de pesquisa. Essa evolução pode ser visualizada no Quadro 2.

\begin{tabular}{|c|c|}
\hline$\overline{\text { DÉCADA }}$ & $\overline{\text { COMENTÁRIOS }}$ \\
\hline DÉCADA DE 1950 & $\begin{array}{l}\text { Nesta década, identificam-se três áreas de pesquisa separadas: } \\
1 \text { - Determinantes psicológi cos do comportamento do consumidor; } \\
2 \text { - Determinantes sociais do comportamento do consumidor; } \\
3 \text { - Tomada de decisão por parte do consumidor. }\end{array}$ \\
\hline DÉCADA DE 1960 & $\begin{array}{l}\text { Um grande número de estudiosos de diferentes áreas começou a } \\
\text { focar esforços para a área de comportamento do consumidor. Linhas } \\
\text { de pesquisas: } \\
1 \text { - Lealdade à marca de produtos de mercearias; } \\
2 \text { - Utilização do método de experimentos no campo do } \\
\text { comportamento do consumidor; } \\
3 \text { - Teoria do risco percebido em comportamento do consumidor; } \\
4 \text { - Teorias compreensivas de comportamento de compra. } \\
\text { Nesta década, foi criada a mais conhecida teoria do comportamento } \\
\text { do consumidor. }\end{array}$ \\
\hline DÉCADA DE 1970 & $\begin{array}{l}\text { Nos anos da década de 1970, houve a consolidação da Association } \\
\text { for Consumer Research (ACR), que foi fundada em 1969. Também } \\
\text { houve a organização do Journal of Consumer Research (JCR) em } \\
\text { 1974. Novas linhas de pesquisas foram desenvolvidas: } \\
\text { 1 - Comportamento de compra industrial; } \\
2 \text { - Estudo do comportamento de consumo de serviços públicos } \\
\text { como cuidados da saúde, transporte, nutrição etc.; } \\
\text { 3 - Comportamento de compra familiar; } \\
\text { 4 - Relacionamento entre atitude e comportamento; } \\
5 \text { - Processamento de informação. }\end{array}$ \\
\hline DÉCADA DE 1980 & $\begin{array}{l}\text { Nesta década, a ênfase das pesquisas recaiu sobre tem as como } \\
\text { rituais e simbolismo, com portamento de fantasia e experimentação e } \\
\text { o impacto da religião no comportamento do consumidor. Também } \\
\text { houve uma corrente contra a mensuração quantitativa nas pesquisas } \\
\text { e uma maior preferência por mais pesquisas de tradição qualitativa. }\end{array}$ \\
\hline DÉCADA DE 1990 & $\begin{array}{l}\text { A partir dos anos 1990, aconteceu o desenvolvimento do marketing } \\
\text { de relacionamento. Assim, passou-se a discutir mais tópicos como } \\
\text { atitudes de consumidores, relações pessoais, tendências de } \\
\text { comportamento, entre outros fatores socioculturais. Os estudos } \\
\text { passaram a se preocupar também com a contextualização do } \\
\text { ambiente. Pode-se assinalar também as melhorias das avaliações } \\
\text { quantitativas proporcionadas pelo avanço tecnológicos e softwares } \\
\text { poderosos de análise de dados. Pode-se afirmar que, nesta década, } \\
\text { houve um crescimento nesse cam po do movimento conhecido } \\
\text { como pós-modernismo - uma forma de investigação que inclui } \\
\text { objetivos e métodos diferentes. }\end{array}$ \\
\hline
\end{tabular}


(continuação Quadro 2)

\begin{tabular}{|l|l|}
\hline \multicolumn{1}{|c|}{ DÉCADA } & \multicolumn{1}{c|}{ COMENTÁR IOS } \\
\hline DÉCADA DE 2000 & $\begin{array}{l}\text { A entrada do novo século estabeleceu uma } \\
\text { perspectiva do comportamento do } \\
\text { consumidor baseada em um "marketing } \\
\text { experiencial", ou seja, a sensação de que os } \\
\text { produtos e serviços proporcionam aos } \\
\text { consumidores experiências sensoriais, } \\
\text { afetivas, cognitivas, físicas e sociais. Assim, } \\
\text { estes passam a ser os novos determinantes } \\
\text { de novos estud os do comportamento do } \\
\text { consumidor. }\end{array}$ \\
\hline
\end{tabular}

Fonte: Sheth, Gardner e Garrett (1988); Vılas Boas, Brito e Sette (2006); tngel, Blackwell e Mınıard (2000).

Quadro 1 - Evolução da escola de comportamento do consumidor.

No Brasil, em virtude da importação de teorias desenvolvidas nos ditos países centrais, vale afirmar que é possível perceber uma defasagem de idéias que retratam uma compreensão tardia da realidade. Ainda que isso possa ser considerado verdadeiro para a área de comportamento do consumidor, também é lícito dizer que esse descompasso vem diminuindo nos últimos anos.

Assim, a partir dessa evolução, cabe afirmar que a área de comportamento do consumidor tem se mostrado como um importante campo de pesquisas na área de marketing. Dessa forma, a análise de artigos nos últimos anos pode indicar tendências de estudos sobre essa temática em nosso país. A próxima seção descreverá os procedimentos metodológicos utilizados neste trabalho.

\section{Metodologia}

O processo metodológico utilizado nesse estudo foi o de desk research, envolvendo o conjunto de artigos científicos sobre o tema comportamento do consumidor, publicados no Brasil nos últimos 10 anos, ou seja, envolvendo a produção acadêmica brasileira de 1997 a 2006. Para isso, foram escolhidos os anais do ENANPAD e os periódicos RAE, RAC e RAUSP. A escolha se deu em virtude de esses anais de congresso e revistas serem classificados como A no sistema Qualis da Capes. Além disso, são considerados os veículos brasileiros mais representativos da produção acadêmica da área de marketing.

Para elaborar o trabalho, utilizou-se o seguinte processo. Todos os artigos da área de marketing foram analisados, separando-se aqueles que tinham como foco central o comportamento do consumidor. Foram excluídos aqueles artigos em duplicidade, ou seja, que haviam sido publicados nos anais do Enanpad e em outro periódico. Nesse caso, foi considerada a publicação no Enanpad. A Tabela 1 apresenta a freqüência dos artigos analisados por ano e publicação. Pode-se perceber que o Enanpad concentra a grande maioria dos artigos $(90,34 \%)$. 
Tabela 1 - Freqüência dos artigos por periódico e ano de publicação

\begin{tabular}{rc|c|c|c|c}
\multicolumn{5}{l|}{ Ano } & \multicolumn{5}{c|}{ Periódicos } & Total \\
\hline & ENANPAD & RAE & RAC & RAUSP & \\
\hline 1997 & 5 & 0 & 0 & 2 & $\mathbf{7}$ \\
\hline 1998 & 11 & 2 & 1 & 0 & $\mathbf{1 4}$ \\
\hline 1999 & 14 & 1 & 0 & 0 & $\mathbf{1 5}$ \\
\hline 2000 & 16 & 1 & 0 & 1 & $\mathbf{1 8}$ \\
\hline 2001 & 17 & 2 & 0 & 0 & $\mathbf{1 9}$ \\
\hline 2002 & 23 & 0 & 1 & 0 & $\mathbf{2 4}$ \\
\hline 2003 & 29 & 3 & 2 & 1 & $\mathbf{3 5}$ \\
2004 & 29 & 1 & 0 & 2 & $\mathbf{3 2}$ \\
2005 & 34 & 0 & 0 & 1 & $\mathbf{3 5}$ \\
2006 & 37 & 0 & 1 & 1 & $\mathbf{3 9}$ \\
Total & $\mathbf{2 1 5}$ & $\mathbf{1 0}$ & $\mathbf{5}$ & $\mathbf{8}$ & $\mathbf{2 3 8}$
\end{tabular}

Fonte: Elaborada pelos autores, com base nos dados da pesquisa.

Em seguida, os artigos foram analisados, utilizando-se um formulário contendo critérios, com o intuito de verificar os elementos básicos de uma investigação, refletindo a qualidade científica de uma pesquisa. As variáveis avaliadas foram adaptadas do guia proposto por Hoppen, Moreau e Lapointe (1997), complementadas pelo estudo de Perin et al. (2000) e pela classificação de métodos de abordagem da pesquisa apresentada por Gonçalves e Meirelles (2004):

- tipo de artigo (empírico ou teórico);

- abordagem da pesquisa (qualitativa ou quantitativa);

- natureza da pesquisa (exploratória, descritiva ou causal);

- segmento de consumidores pesquisados;

- metodologia (survey, experimentos ou de base qualitativa);

- tema do artigo;

- embasamento conceitual (teorias e conceitos de base, objetivos, questão de pesquisa, hipóteses ou pressupostos de base);

- desenho de pesquisa (modelo de pesquisa, operacionalização das variáveis);

- coleta de dados (tipos de dados, forma de coleta de dados, tipo de amostra);

- validade dos construtos;

- tipo de análise de dados;

- apresentação dos resultados (limites do estudo, recomendações para pesquisas futuras e recomendações aplicadas).

Vale ressaltar que o formulário para os artigos de base qualitativa teve alguns pontos retirados, em virtude das peculiaridades deste tipo de trabalho. É importante relatar também que os artigos teóricos não foram analisados com base nessas variáveis.

e Excel.

Os dados, após serem coletados, foram tabulados e processados pelos softwares SPSS 


\section{Apresentação e análise dos resultados}

Verifica-se, pela Tabela 2, a freqüência marcante (82,35\%) de artigos empíricos na área do comportamento do consumidor no período analisado.

Tabela 2 - Freqüência dos artigos por tipo

\begin{tabular}{|l|c|c|}
\hline \multicolumn{1}{|c|}{ Tipo do artigo } & N. & Perc. \\
\hline Teórico & 42 & $17,65 \%$ \\
\hline Empírico & 196 & $82,35 \%$ \\
\hline Total & $\mathbf{2 3 8}$ & $\mathbf{1 0 0 , 0 0 \%}$ \\
\hline
\end{tabular}

Fonte: Elaborada pelos autores, com base nos dados da pesquisa.

Ao se analisar a Tabela 3, dos artigos considerados como empíricos, verifica-se a predominância de pesquisas de natureza quantitativa (aproximadamente 80\%). Esse dado é importante para sinalizar a adoção de referenciais eminentemente positivistas para a pesquisa do consumidor.

Tabela 3 - Freqüência dos artigos por abordagem de pesquisa

\begin{tabular}{l|c|c}
\hline Abordagem & N. & Perc. \\
\hline Qualitativa & 39 & $19,90 \%$ \\
\hline Quantitativa & 157 & $80,10 \%$ \\
\hline Total & 196 & $100,00 \%$ \\
\hline
\end{tabular}

Fonte: Elaborada pelos autores, com base nos dados da pesquisa.

Visualiza-se, na Tabela 4, que a grande maioria dos trabalhos (cerca de 71\%) da área do comportamento do consumidor no Brasil nos últimos 10 anos utilizou o método de survey. Apenas $9,18 \%$ dos artigos apontaram o método de experimento e cerca de $20 \%$ utilizaram-se de metodologias qualitativas.

Com relação à natureza de uma pesquisa, segundo Churchill Jr. (1999), uma pesquisa exploratória tem como escopo aprofundar conceitos preliminares, objetivando a geração de idéias, e desenvolver hipóteses e proposições que irão permitir a realização de outras pesquisas. Já as pesquisas descritivas buscam determinar a freqüência com que algo ocorre ou a relação entre duas ou mais variáveis, sendo normalmente guiadas por uma hipótese inicial. As pesquisas de natureza causal, por sua vez, têm como finalidade o teste de uma teoria e suas relações de causa e efeito. 
Tabela 4 - Freqüência dos artigos por método

\begin{tabular}{l|c|c}
\hline Métodos & N. & Perc. \\
\hline Survey & 139 & $70,92 \%$ \\
\hline Experimentos & 18 & $9,18 \%$ \\
\hline Qualitativas & 39 & $19,90 \%$ \\
Total & $\mathbf{1 9 6}$ & $\mathbf{1 0 0 , 0 0 \%}$ \\
\hline
\end{tabular}

Fonte: Elaborada pelos autores, com base nos dados da pesquisa.

Conforme pode ser visualizado na Tabela 5, dos 196 artigos considerados como empíricos, destaca-se a natureza descritiva das pesquisas realizadas na área de comportamento do consumidor $(60,71 \%)$.

Tabela 5 - Freqüência dos artigos por natureza de pesquisa

\begin{tabular}{l|c|c}
\hline Natureza & N. & Perc. \\
\hline Exploratórias & 50 & $25,51 \%$ \\
\hline Descritivas & 119 & $60,71 \%$ \\
\hline Causais & 27 & $13,78 \%$ \\
\hline Total & 196 & $\mathbf{1 0 0 , 0 0 \%}$ \\
\hline
\end{tabular}

Fonte: Elaborada pelos autores, com base nos dados da pesquisa.

Conforme pode ser visualizado na Tabela 6, dos 157 artigos quantitativos, percebese um percentual considerável da utilização de estudantes como segmento de consumidores pesquisado (quase $30 \%$ de todos os artigos).

No que tange ao tema relacionado ao comportamento do consumidor discutido nos artigos, não foi possível elaborar uma tabela sucinta dos resultados, em face da não convergência dos temas. Contudo, as principais temáticas abordadas foram: satisfação do consumidor, comportamento do consumidor no comércio eletrônico e comportamento do consumidor para produtos e serviços específicos.

Tabela 6 - Freqüência dos artigos por segmento de consumidores pesquisados

\begin{tabular}{l|c|c}
\hline & N. & Perc. \\
\hline Estudantes & 46 & $29,30 \%$ \\
\hline Internautas & 14 & $8,92 \%$ \\
Outros & 97 & $61,78 \%$ \\
\hline Total & 157 & $100,00 \%$
\end{tabular}

Fonte: Elaborada pelos autores, com base nos dados da pesquisa.

Nos próximos tópicos, serão apresentadas as análises dos artigos, baseadas em pesquisas do tipo survey, do tipo experimentais e pesquisas qualitativas. 


\subsection{Análise dos artigos baseados em pesquisas do tipo survey}

A Tabela 7 apresenta sinteticamente os resultados da análise dos 139 artigos do tipo survey. Alguns pontos podem ser ressaltados: superficial;

- Mais de $70 \%$ dos artigos apresentaram as teorias e conceitos de base de forma

- Mais da metade dos artigos não apresentou questões da pesquisa e 48,20\% não apresentaram o modelo de pesquisa;

- Mais de 55\% dos artigos não apresentaram a operacionalização das variáveis;

- 71,22\% dos artigos utilizaram-se da entrevista pessoal como forma de coleta de dados;

- Percebeu-se um predomínio das amostras não-probabilísticas (78,72\%) das amostragens;

- 45,32\% das pesquisas não apresentaram os testes de confiabilidade;

- 46,04\% das pesquisas não incluíram nos artigos os testes de validade;

- Verificou-se a predominância da utilização de análises descritivas multivariadas na análise dos dados (cerca de 62\%);

- Com relação à apresentação dos resultados, constatou-se que boa parte dos artigos não apresentou limites dos estudos (58,99\%), recomendações para pesquisas futuras $(46,76 \%)$ e recomendações aplicadas (54,68\%).

Tabela 7 - Síntese da análise dos artigos baseados em pesquisas do tipo survey

\begin{tabular}{ll|cc}
\hline & & N & Perc. \\
\hline \multirow{3}{*}{ Teorias e conceitos de base } & Aprofundado & 41 & $29,50 \%$ \\
& Superficial & 98 & $70,50 \%$ \\
& Ausente & 0 & $0,00 \%$ \\
\hline Objetivos & Sim & 128 & $92,09 \%$ \\
Questão de pesquisa, hipóteses & Não & 11 & $7,91 \%$ \\
ou pressupostos de base & Sim & 64 & $46,04 \%$ \\
& Não & 75 & $53,96 \%$ \\
Modelo de pesquisa & Sim - com clareza & 45 & $32,37 \%$ \\
& Sim- pouca clareza & 27 & $19,42 \%$ \\
Operacionalização das variáveis & Não & 67 & $48,20 \%$ \\
& Sim & 62 & $44,60 \%$ \\
& Não & 77 & $55,40 \%$ \\
Tipos de dados & Primários & 134 & $96,40 \%$ \\
& Secundários & 4 & $2,88 \%$ \\
Forma de coleta de dados & Ambos & 1 & $0,72 \%$ \\
& Entrevista pessoal & 99 & $71,22 \%$ \\
& Correio & 10 & $7,19 \%$ \\
& Eletrônico & 16 & $11,51 \%$ \\
& Telefone & 7 & $5,04 \%$ \\
& Outro & 7 & $5,04 \%$ \\
\hline
\end{tabular}


(continuação Tabela 7)

\begin{tabular}{ll|cc}
\hline & & N & Perc. \\
\hline \multirow{2}{*}{ Confiabilidade } & Ausente & 63 & $45,32 \%$ \\
& Cronbach & 70 & $50,36 \%$ \\
& Outro & 6 & $4,32 \%$ \\
\hline \multirow{2}{*}{ Validade } & Ausente & 64 & $46,04 \%$ \\
& Convergente/Discriminante & 73 & $52,52 \%$ \\
& Outro & 2 & $1,44 \%$ \\
\hline \multirow{2}{*}{ Tipo de análise de dados } & Descritiva univariada & 10 & $7,19 \%$ \\
& Descritiva multivariada & 86 & $61,87 \%$ \\
& Equações estruturais & 39 & $28,06 \%$ \\
\hline Limites do estudo & Outros & 4 & $2,88 \%$ \\
\hline Recomendações para pesquisas & Sim & 57 & $41,01 \%$ \\
futuras & Não & 82 & $58,99 \%$ \\
\hline Recom endações aplicadas & Sim & 65 & $53,24 \%$ \\
& Não & 63 & $46,76 \%$ \\
& Sim & 76 & $54,32 \%$ \\
\hline
\end{tabular}

Fonte: Elaborada pelos autores, com base nos dados da pesquisa.

\subsection{Análise dos artigos baseados em pesquisas do tipo survey}

A Tabela 8 apresenta sinteticamente os resultados da análise dos 18 artigos baseados em pesquisas experimentais.

Tabela 8 - Síntese da análise dos artigos baseados em pesquisas experimentais

\begin{tabular}{l|l|c|c}
\hline & & N & Perc. \\
\hline \multirow{3}{*}{ Teorias e conceitos de base } & Aprofundado & 9 & $50,00 \%$ \\
& Superficial & 9 & $50,00 \%$ \\
& Ausente & 0 & $0,00 \%$ \\
\hline Objetivos & Sim & 18 & $100,00 \%$ \\
Questão de pesquisa, hipóteses ou & Não & 0 & $0,00 \%$ \\
pressupostos de base & Sim & 16 & $88,89 \%$ \\
Modelo de pesquisa & Não & 2 & $11,11 \%$ \\
Operacionalização das variáveis & Sim - com clareza & 8 & $44,44 \%$ \\
Tipos de dados & Sim- pouca clareza & 3 & $16,67 \%$ \\
& Não & 7 & $38,89 \%$ \\
Forma de coleta de dad os & Sim & 9 & $50,00 \%$ \\
& Não & 9 & $50,00 \%$ \\
& Primários & 0 & 18 \\
& Secundários & 0 & $0,00 \%$ \\
& Ambos & 15 & $83,00 \%$ \\
& Entrevista pessoal & 0 & $0,00 \%$ \\
& Correio & 2 & $11,11 \%$ \\
& Eletrônico & 1 & $5,56 \%$ \\
& Telefone & 0 & $0,00 \%$ \\
\hline
\end{tabular}


(continuação Tabela 8)

\begin{tabular}{l|l|c|c}
\hline & & N & Perc. \\
\hline \multirow{3}{*}{ Tipo de amostra } & Probabilística & 0 & $0,00 \%$ \\
& Não-probabilística & 18 & $100,00 \%$ \\
& Não declarado & 0 & $0,00 \%$ \\
\hline \multirow{3}{*}{ Confiabilidade } & Ausente & 5 & $27,78 \%$ \\
& Cronbach & 13 & $72,22 \%$ \\
& Outro & 0 & $0,00 \%$ \\
\hline \multirow{3}{*}{ Validade } & Ausente & 6 & $33,33 \%$ \\
& Convergente/Discriminante & 12 & $66,67 \%$ \\
Tipo de análise de dados & Outro & 0 & $0,00 \%$ \\
& Descritiva univariada & 1 & $5,56 \%$ \\
& Descritiva multivariada & 12 & $66,67 \%$ \\
& Equações estruturais & 5 & $27,78 \%$ \\
& Outros & 0 & $0,00 \%$ \\
& Sim & 11 & $61,11 \%$ \\
Recomendações para pesquisas & Não & 7 & $38,89 \%$ \\
\hline Recomendações aplicadas & Sim & 9 & $50,00 \%$ \\
& Não & 9 & $50,00 \%$ \\
\hline
\end{tabular}

Fonte: Elaborada pelos autores, com base nos dados da pesquisa.

Vale destacar os seguintes pontos:

- Todos os artigos desse tipo apresentaram os objetivos da pesquisa e quase $90 \%$ deles enunciaram a questão da pesquisa;

- 38,89\% não apresentaram o modelo de pesquisa utilizado;

- Metade dos artigos não apresentou a operacionalização das variáveis;

- 83,33\% utilizaram a entrevista pessoal como principal forma de coleta de dados;

- A totalidade dos trabalhos lançou mão das amostras não-probabilísticas;

- 27,78\% dos artigos não apresentaram os testes de confiabilidade e 33,33 não incluíram os testes de validade;

- Observou-se que grande parte dos trabalhos (66,67\%) analisou os dados por meio de técnicas estatísticas multivariadas;

- Com relação à apresentação dos resultados, constatou-se que 38,89\% não apresentaram limites dos estudos, $50 \%$ não discutiram as recomendações para pesquisas futuras e 55,56\% não citaram recomendações aplicadas da pesquisa.

Vale ressaltar que, a despeito do número de artigos, numa comparação mais detalhada entre a análise dos artigos baseados em pesquisas do tipo survey e dos artigos do tipo experimentais, verificou-se uma ligeira qualidade superior dos últimos nos pontos analisados.

\subsection{Análise dos artigos baseados em pesquisas qualitativas}

A Tabela 9 apresenta os resultados da análise dos 39 artigos baseados em pesquisas qualitativas. Podem ser destacados os seguintes pontos: 
- Quase metade dos artigos (48,72\%) apresentou as teorias e conceitos de base de forma aprofundada;

- 92,31\% descreveram os objetivos do estudo;

- A grande maioria dos artigos de base qualitativa não apresentou as questões de pesquisa $(84,62 \%)$, nem modelos de pesquisa $(76,92 \%)$;

- Com relação ao tipo de metodologia, pode-se afirmar que não houve convergência das metodologias, porém, com ligeiro destaque, podem ser citados os estudos de caso;

- Como na análise de outros tipos de artigos, percebeu-se que a quase totalidade dos trabalhos utilizou-se de dados primários;

- Como ferramentas de coleta de dados, destacaram-se as entrevistas não-estruturadas e semi-estruturadas, sendo escolhidas por aproximadamente $70 \%$ dos pesquisadores da área;

- A análise de conteúdo e a análise de discurso foram as mais utilizadas para análise dos dados, correspondendo a quase $85 \%$ da totalidade dos artigos analisados;

- No que tange às variáveis relativas à apresentação dos resultados, notou-se que boa parte dos artigos não incluiu limites do estudo (87,18\%), não apresentou recomendações para pesquisas futuras $(56,41 \%)$, nem descreveu recomendações aplicadas $(64,10 \%)$.

Tabela 9 - Síntese da análise dos artigos baseados em pesquisas qualitativas

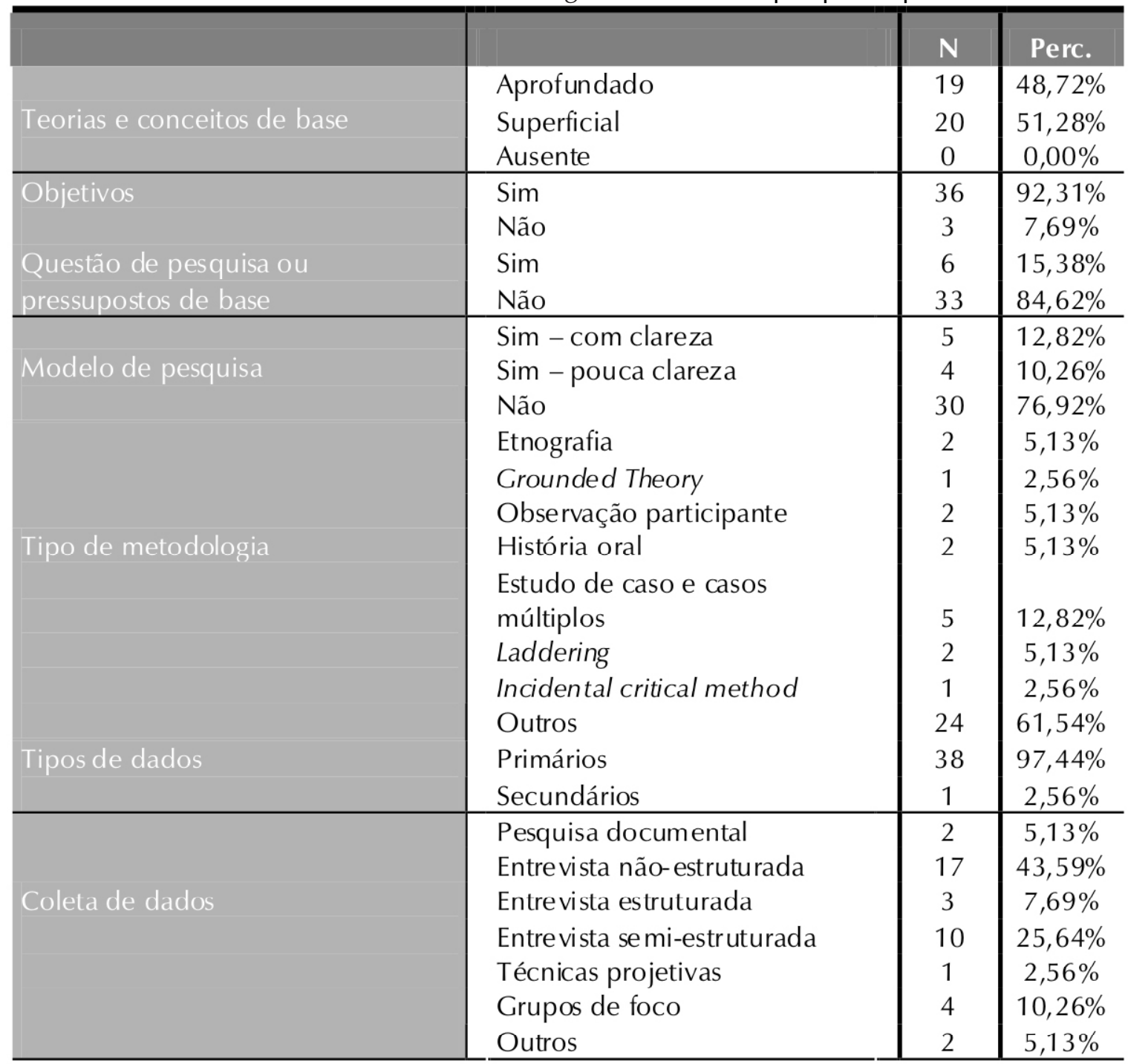


(continuação Tabela 9)

\begin{tabular}{l|l|c|c}
\hline & & N & Perc. \\
\cline { 1 - 3 } Análise de dados & Análise de conteúdo & 19 & $48,72 \%$ \\
& Análise de discurso & 14 & $35,90 \%$ \\
& Análise de significado & 2 & $5,13 \%$ \\
& Outros & 4 & $10,26 \%$ \\
& Sim & 5 & $12,82 \%$ \\
\hline Recomites do estudo & Não & 34 & $87,18 \%$ \\
futuras & Sim & 17 & $43,59 \%$ \\
Recomões para pesquisas & & & \\
& Não & 22 & $56,41 \%$ \\
& Sim & 14 & $35,90 \%$ \\
& Não & 25 & $64,10 \%$ \\
\hline
\end{tabular}

Fonte: Elaborada pelos autores, com base nos dados da pesquisa.

\section{Considerações e reflexões finais}

Com base nos resultados descritos na seção anterior, a título de conclusão, alguns pontos devem ser assinalados. Em primeiro lugar, é evidente o crescimento da área do comportamento do consumidor nas últimas décadas. Novos campos de pesquisas têm sido criados, outras disciplinas de áreas diversas do conhecimento, como Antropologia, História, Sociologia e Estatística, vêm contribuindo de forma decisiva no desenvolvimento da área, e o número de trabalhos tanto em periódicos nacionais e internacionais vêm aumentando sensivelmente. A não convergência de temas presentes nos artigos pesquisados parece corroborar com essa constatação.

Com a análise dos artigos relacionados ao comportamento do consumidor publicados nos últimos dez anos nos principais veículos de divulgação da produção acadêmica em marketing no Brasil, pôde-se ter uma visão da tendência das publicações da área. Conforme relatado nos resultados, pode-se afirmar que um dos principais resultados do trabalho remete à questão da tendência de trabalhos com base empírica, de abordagem positivista e utilizando métodos e técnicas quantitativistas tradicionais de pesquisa e coleta de dados.

Com base nessas constatações, pode-se fazer alguns questionamentos: a baixa publicação de artigos com base teórica seria decorrente de uma dificuldade de teorização por parte dos autores ou uma recusa dos periódicos em publicar esses trabalhos? Por que a insistência em se utilizar métodos e técnicas tradicionais de pesquisas, visto que, atualmente, o rol de possibilidades de novas metodologias aumenta consideravelmente? Não seria hora de propor novas metodologias, visando a ampliar o entendimento dos fenômenos do comportamento do consumidor? Por que a grande maioria dos trabalhos se baseia no paradigma positivista? Também não seria hora de se propor novas abordagens de cunho fenomenológico ou interpretativista, a fim de se investigar melhor o comportamento dos consumidores a partir de outra perspectiva?

Conforme bem enfatizado por Casotti (1999), o estudo do comportamento do consumidor parece necessitar de outras abordagens e metodologias de pesquisa que não se apresentem apenas como um processo linear e estático, pelo contrário, possibilitem interações e construções em torno de temas que têm a diversidade, a multidisciplinaridade e o conflito como peculiaridades básicas. No mesmo sentido, Zaltman (2000) reconhece a importância de trabalhos de outras áreas serem incorporados ao campo de conhecimento do comportamento 
do consumidor. Ademais, esse autor identifica a necessidade de serem utilizadas abordagens multidisciplinares, uma vez que o comportamento do consumidor oferece uma riqueza enorme de problemáticas que, não raramente, não podem ser captadas por modelos criados a partir de uma única abordagem ou disciplina.

Não menos importante é a constatação de que podem ser citadas inúmeras situações em que as abordagens tradicionais de pesquisa não conseguem explicar os fenômenos ou o fazem de forma superficial ou equivocada, tais como: relacionamento interativo entre os clientes e os vendedores, a influência das condições físicas do ambiente nos processos de compra, o comportamento de compra socialmente responsável por parte de um contingente de consumidores, o comportamento de compra baseado em experiências, entre outros.

Sendo assim, outras metodologias diferentes das tradicionais quantitativas e funcionalistas e próximas das fenomenológicas e interpretativas poderiam ser utilizadas para ampliar o leque de opções do pesquisador da área. Por exemplo, podem ser citadas a etnografia, a fotoetnografia, a netnografia, a metodologia reflexiva, a grounded theory, as técnicas projetivas, a técnica de autodriving, entre diversas outras. Vale ressaltar aqui que não se trata de abandonar a pesquisa quantitativa, mas sim buscar combinações de metodologias e técnicas, a fim de melhorar a qualidade das pesquisas.

De volta aos resultados da análise, outros pontos são dignos de maiores discussões. Verificou-se uma baixa consideração, em média, nos vários tipos de artigos analisados, aos seguintes elementos metodológicos: questões de pesquisa, hipóteses ou pressupostos de base, modelo de pesquisa, operacionalização das variáveis, limites do estudo e recomendações para pesquisas futuras, o que pode sinalizar uma pouca preocupação dos pesquisadores com aspectos relevantes da qualidade metodológica. Percebe-se também um alto percentual de pesquisas de natureza descritiva $(60,71 \%)$ em relação às pesquisas de natureza exploratória $(25,51 \%)$ e causal (13,78\%). Além disso, percebe-se uma predominância, nos artigos quantitativos, da utilização de amostras não-probabilísticas. No mesmo sentido, ficou óbvia a predominância do uso de análises estatísticas multivariadas, ainda que se possa perceber uma tendência de maior preferência pelas técnicas de modelagem de equações estruturais, principalmente a partir do ano de 2000, quando, em média, 30\% dos artigos passaram a utilizar essa técnica.

Contudo, deve ser ressaltado que os resultados da análise dos artigos do tipo experimentais apresentaram resultados superiores aos do tipo survey com relação a diversas variáveis analisadas. Ademais, pode-se dizer também que, quando comparados ao estudo conduzido por Perin et al. (2000) relativos aos artigos de survey na década de 1990, os indicadores relativos à confiabilidade e validade se apresentam muito melhores.

Cabe, nesse ponto, destacar algumas limitações do trabalho. Em virtude do "corte" feito nas edições dos últimos dez anos dos periódicos, é importante ressaltar que isso pode ter levado a criação de vieses nas conclusões. Outra limitação reside no fato de que, ainda que se tenha elaborado um roteiro de questões com critérios claros para orientar a análise, muitas vezes, os artigos apresentaram uma descrição confusa ou incompleta da metodologia utilizada, o que dificultou a classificação dos trabalhos. Ademais, é interessante comentar que foi desconsiderada, neste trabalho, uma eventual evolução longitudinal da produção científica analisada.

Seria útil também propor a continuação deste trabalho, incorporando a análise de artigos numa base maior de tempo, tanto nos periódicos já pesquisados quanto em outras importantes journals internacionais como, por exemplo, o Journal of Consumer Research, o Journal of Marketing Research e o Journal of Consumer Marketing entre outros. Dessa forma, a comunidade acadêmica da área de marketing poderia ter um mapeamento mais detalhado e, conseqüentemente, mais fiel dos estudos e dos caminhos que vem sendo percorridos pelos 
pesquisadores da área.

Por fim, mas não menos importante, vale afirmar que alguns pontos podem ser assinalados com vistas a contribuir para a melhoria da produção científica do comportamento do consumidor no Brasil. Seja nas instituições de ensino e pesquisa, seja nos diversos programas de pós-graduação em Administração, linhas de pesquisas com foco na temática do estudo do consumidor deveriam ser criadas ou mais bem organizadas. Da mesma forma, pesquisas cumulativas deveriam ser planejadas e executadas com o escopo de se aprofundar nos temas estudados. Por sua vez, os pesquisadores da área deveriam zelar por um maior rigor metodológico dos estudos realizados. Outra contribuição poderia vir da organização de eventos (seminários, congressos, workshops, encontros etc.), assim como a criação de publicações que serviriam de veículos de divulgação do conhecimento local e para a consolidação dessa área tão desafiadora e instigante que é a do comportamento do consumidor. 


\section{Referências bibliográficas}

BERTERO, C. O., KEINERT, T. M. M. A evolução da análise organizacional no Brasil (1961-93). Revista de Administração de Empresas, v. 34, n. 3, p. 81-90, 1994.

CASOTTI, L. O que é a pesquisa do consumidor? Reflexões geradas a partir de um problema prático. In: ENCONTRO NACIONAL DE PROGRAMAS DE PÓS GRADUAÇÃO EM ADMINISTRAÇÃO, 23., 1999, Foz do Iguaçu. Anais... Foz do Iguaçu: ANPAD, 1999. CDROOM.

CHURCHILL JR., G. A. Marketing research: methodological foundation. Orlando: Dryden, 1999.

ENGEL, J. F. ; BLACKWELL, R. D.; MINIARD, P. W. Comportamento do consumidor. 8. ed. Rio de Janeiro: Livros Técnicos e Científicos, 2000.

FARIA, P. C. N. et al. Mapeamento, análise e classificação dos trabalhos acadêmicos de marketing nos ENANPADs de 2000 a 2005. In: ENCONTRO DE MARKETING, 2., 2006, Rio de Janeiro. Anais... Rio de Janeiro: ANPAD, 2006. CD-ROOM.

GONÇALVES. C. A.; MEIRELLES, A. M. Projetos e relatórios de pesquisa em administração. São Paulo: Atlas, 2004.

HOPPEN, N.; MEIRELLES, F.S. Sistemas de informação: um panorama da pesquisa científica entre 1990 e 2003. Revista de Administração de Empresas, v. 45, n.1, p. 24-36, 2005.

HOPPEN, N.; MOREAU, E.; LAPOINTE, L. Avaliação de artigos de pesquisa em sistemas de informação: proposta de um guia. In: ENCONTRO NACIONAL DE PROGRAMAS DE PÓS GRADUAÇÃO EM ADMINISTRAÇÃO, 24., 1997, Angra dos Reis. Anais... Angra dos Reis: ANPAD, 1997. CD-ROOM.

LEAL, R.; OLIVEIRA, J.; SOLURI, A. Perfil da pesquisa em finanças no Brasil. Revista de Administração de Empresas, v. 43, n.1, p. 91 104, 2003.
MACHADO-DA-SILVA, C.; CUNHA, V. C. da; AMBONI, N. Organizações: o estado da arte da produção acadêmica no Brasil. In: ENCONTRO NACIONAL DE PROGRAMAS DE PÓS GRADUAÇÃO EM ADMINISTRAÇÃO, 14., 1990, Belo Horizonte. Anais... Belo Horizonte: ANPAD, v. 6, p.11-28, 1990.

PERIN, M. G. et al. A perspectiva survey em artigos de marketing nos ENANPADs da década de 90. In: ENCONTRO NACIONAL DE PROGRAMAS DE PÓS GRADUAÇÃO EM ADMINISTRAÇÃO, 24., 1999, Florianópolis. Anais... Florianópolis: ANPAD, 2000. CDROOM.

SHETH, J. N.; GARDNER, D. M.; GARRETT, D. E. Marketing theory: evolution and evaluation. New York: John Wiley \& Sons, 1988.

RODRIGUES, S. B.; CARRIERI, A. Estudos organizacionais: a tradição anglo-saxônica no Brasil. In: RODRIGUES, S. B.; CUNHA, M. P. (Orgs.). Estudos organizacionais: novas perspectivas para a administração de empresas - uma coletânea luso-brasileira. São Paulo: Iglu, 2000. p 21-42.

TONELLI, M. et al. Produção acadêmica em recursos humanos no Brasil: 1991-2000. Revista de Administração de Empresas, v. 43, n.1, p. 105-122, 2003.

VERGARA, S. C.;, CARVALHO JR., D. de S. Nacionalidade dos autores referenciados na literatura brasileira sobre organizações. In: ENCONTRO NACIONAL DE PROGRAMAS DE PÓS GRADUAÇÃO EM ADMINISTRAÇÃO, 19., 1995, João Pessoa. Anais... João Pessoa: ANPAD, v. 6, p.169-188, 1995.

VIEIRA, F. G. D. Por quem os sinos dobram? Uma análise da publicação científica na área de marketing do ENANPAD. In: ENCONTRO NACIONAL DE PROGRAMAS DE PÓS GRADUAÇÃO EM ADMINISTRAÇÃO, 22., 1998, Foz do Iguaçu. Anais... Foz do Iguaçu: ANPAD, 1998. CD-ROOM. 
- Ações empresariais e prioridades de

pesquisa em marketing: tendências no Brasil e no

mundo, segundo a percepção dos acadêmicos

brasileiros. In: ENCONTRO NACIONAL DE

PROGRAMAS DE PÓS GRADUAÇÃO EM

ADMINISTRAÇÃO, 23., 1999, Foz do Iguaçu.

Anais... Foz do Iguaçu: ANPAD, 1999. CD-

ROOM.

Panorama acadêmico-científico e

temáticas de estudos de marketing no Brasil.

In: ENCONTRO NACIONAL DE PROGRAMAS

DE PÓS GRADUAÇÃO EM ADMINISTRAÇÃO,

24., 1999, Florianópolis. Anais... Florianópolis:

ANPAD, 2000. CD-ROOM.

VILAS BOAS, L. H. B.; BRITO, M. J.; SETTE, R.

S. Perspectivas da Antropologia no estudo do

comportamento do consumidor: contribuições para o desenvolvimento da teoria e pesquisa de marketing. Revista Eletrônica de Administração, ed. 50, v. 12, n. 2, mar./abr. 2006.

ZALTMAN, G. Consumer researchers: take a hike! Journal of Consumer Research, v. 26, p. 423-428, Mar. 2000. 